\title{
AN AZIMUTHAL DYNAMO WAVE IN SPHERICAL SHELL CONVECTION
}

\author{
Elizabeth Cole ${ }^{1}$, Petri J. KäPylä̈ ${ }^{1,2}$, MaArit J. Mantere ${ }^{1,3}$, and Axel Brandenburg ${ }^{2,4}$ \\ ${ }^{1}$ Physics Department, Gustaf Hällströmin katu 2a, P.O. Box 64, FI-00014 University of Helsinki, Finland; elizabeth.cole@ helsinki.fi \\ ${ }^{2}$ NORDITA, KTH Royal Institute of Technology and Stockholm University, Roslagstullsbacken 23, SE-10691 Stockholm, Sweden \\ ${ }^{3}$ Department of Information and Computer Science, Aalto University, P.O. Box 15400, FI-00076 Aalto, Finland \\ ${ }^{4}$ Department of Astronomy, AlbaNova University Center, Stockholm University, SE-10691 Stockholm, Sweden \\ Received 2013 September 26; accepted 2013 November 25; published 2013 December 16
}

\begin{abstract}
We report the discovery of an azimuthal dynamo wave of a low-order $(m=1)$ mode in direct numerical simulations (DNS) of turbulent convection in spherical shells. Such waves are predicted by mean-field dynamo theory and have been obtained previously in mean-field models. An azimuthal dynamo wave has been proposed as a possible explanation for the persistent drifts of spots observed on several rapidly rotating stars, as revealed through photometry and Doppler imaging. However, this has been judged unlikely because evidence for such waves from DNS has been lacking. Here we present DNS of large-scale magnetic fields showing a retrograde $m=1$ mode. Its pattern speed is nearly independent of latitude and does not reflect the speed of the differential rotation at any depth. The extrema of magnetic $m=1$ structures coincide reasonably well with the maxima of $m=2$ structures of the temperature. These results provide direct support for the observed drifts being due to an azimuthal dynamo wave.
\end{abstract}

Key words: convection - dynamo - magnetohydrodynamics (MHD) - stars: activity - stars: late-type - turbulence

Online-only material: color figures

\section{INTRODUCTION}

The solar large-scale magnetic field is mostly axisymmetric and exhibits a dynamo wave propagating from mid-latitudes toward the equator. The solar cycle is often explained in terms of $\alpha \Omega$ dynamo models based on mean-field theory where the poloidal field is regenerated via cyclonic turbulence $(\alpha$-effect) and the toroidal field through differential rotation $(\Omega$-effect), see, e.g., Ossendrijver (2003). For faster rotation, the $\alpha$-effect becomes strongly anisotropic (Rüdiger 1978) while its magnitude is less strongly suppressed than turbulent diffusivity (Käpylä et al. 2009). At the same time, differential rotation is also quenched (e.g., Kitchatinov \& Rüdiger 1999), which enables non-axisymmetric modes to dominate. Thus, in more rapidly rotating stars, the large-scale magnetic field is expected to become more non-axisymmetric (Rädler et al. 1990; Moss et al. 1995).

Recent numerical simulations have reached a level of sophistication where they have been able to produce oscillatory large-scale magnetic fields (e.g., Ghizaru et al. 2010; Käpylä et al. 2010; Brown et al. 2011; Nelson et al. 2013) and in some cases equatorward migration as in the Sun (Käpylä et al. 2012, 2013; Warnecke et al. 2013; Augustson et al. 2013). Furthermore, as the rotation rate is increased, non-axisymmetric large-scale fields are obtained (Goudard \& Dormy 2008; Gastine et al. 2012; Käpylä et al. 2013), as expected from meanfield dynamo theory.

Observational results from photometry, spectroscopy and spectropolarimetry show a similar trend for rapid rotators with high levels of magnetic activity, manifested through extended high-latitude starspots that have a predominantly nonaxisymmetric longitudinal distribution (e.g., Berdyugina \& Tuominen 1998; Kochukhov et al. 2013). The most often deduced configuration consists of two active longitudes with alternating levels of activity. This is referred to as the flip-flop phenomenon after the original work of Jetsu et al. (1993) in the context of phase jumps seen on the single giant star FK Com.
With the accumulation of observational data, it has become evident that the flip-flopping does not occur periodically (see, e.g., Korhonen et al. 2007; Hackman et al. 2013). Moreover, in almost all cases the phase behavior of the active longitude system shows disrupted linear trends in the rotational frame of reference, i.e., the system is usually not rotating with the same speed as the stellar surface. One of the most prominent examples of this is the primary component of the RS CVn binary system II Peg, where a drift pattern persistent over a $10 \mathrm{yr}$ epoch has been reported (Hackman et al. 2012; Lindborg et al. 2013). Such drifts are traditionally not explained by the presence of an azimuthal dynamo wave, but by surface differential rotation causing the spots to move with different speeds as their latitude changes, analogously to the Sun. In some cases, such as FK Com, the changing angular velocity can clearly be related to changes in spot latitudes (see, e.g., Korhonen et al. 2007). This picture, however, seems less evident in II Peg, in which no major changes in spot latitudes can be observed. However, the magnetic structures move in the prograde direction with respect to a rotating frame (Lindborg et al. 2013).

The idea that spots reflect the motion of the gas seems quite straightforward, but there are various reasons why the pattern speed associated with spots can be different from that of the gas. Sunspots exhibit a prograde motion, which is often associated with sunspots being anchored at some other depth where they match the local speed of the gas (e.g., Pulkkinen \& Tuominen 1998). However, in the Sun, magnetic tracers usually move faster than the gas (Gizon et al. 2003), which can be explained as a property of hexagon-like convection cells in the presence of rotation (Busse 2004, 2007), but it might also be related to the near-surface shear layer in the Sun (Green \& Kosovichev 2006; Brandenburg 2007). However, these are local considerations, so we should still expect the pattern speed to reflect the equatorial acceleration near the equator. By contrast, in linear dynamo theory, a nonaxisymmetric dynamo mode always rotates like a rigid body (Rädler 1986). Depending on model details and the sign of the $\alpha$ effect, both prograde and retrograde rotation 
of the pattern is possible. Rädler (1986) discuss the so-called westward drift of Earth's magnetic field in the context of the geodynamo. Rigidly rotating patterns also occur in the nonlinear regime (Rädler et al. 1990). Thus, we should expect that dynamo patterns would not bear any information about latitudinal differential rotation.

Here we report on simulations of rapidly rotating turbulent convection that exhibit large-scale non-axisymmetric magnetic fields with azimuthal dynamo waves. We show that the pattern speed of non-axisymmetric structures is essentially constant, as expected from mean-field theory.

\section{THE MODEL}

Our model is similar to that of Käpylä et al. (2012, 2013). We model a shell in spherical polar coordinates, where $(r, \theta, \phi)$ denote radius, colatitude, and longitude. Here we model a shell $r_{0} \leqslant r \leqslant R, \theta_{0} \leqslant \theta \leqslant \pi-\theta_{0}$, and $0 \leqslant \phi \leqslant \phi_{0}$, where $r_{0}=0.7 R, \theta_{0}=\pi / 12, \phi_{0}=2 \pi$ and $R$ is the radius of the star. We solve the compressible hydromagnetic equations,

$$
\begin{gathered}
\frac{\partial \boldsymbol{A}}{\partial t}=\boldsymbol{u} \times \boldsymbol{B}-\mu_{0} \eta \boldsymbol{J}, \\
\frac{D \ln \rho}{D t}=-\boldsymbol{\nabla} \cdot \boldsymbol{u}, \\
\frac{D \boldsymbol{u}}{D t}=\boldsymbol{g}-2 \boldsymbol{\Omega}_{0} \times \boldsymbol{u}+\frac{1}{\rho}(\boldsymbol{J} \times \boldsymbol{B}-\nabla p+\nabla \cdot 2 \nu \rho \mathbf{S}), \\
T \frac{D s}{D t}=\frac{1}{\rho}\left[-\nabla \cdot\left(\boldsymbol{F}^{\mathrm{rad}}+\boldsymbol{F}^{\mathrm{SGS}}\right)+\mu_{0} \eta \boldsymbol{J}^{2}\right]+2 \nu \mathbf{S}^{2},
\end{gathered}
$$

where $\boldsymbol{A}$ is the magnetic vector potential, $\boldsymbol{u}$ is the velocity, $\boldsymbol{B}=\boldsymbol{\nabla} \times \boldsymbol{A}$ is the magnetic field, $\boldsymbol{J}=\mu_{0}^{-1} \nabla \times \boldsymbol{B}$ is the current density, $\mu_{0}$ is the vacuum permeability, $D / D t=\partial / \partial t+\boldsymbol{u} \cdot \boldsymbol{\nabla}$ is the advective time derivative, $\rho$ is the density, $\boldsymbol{g}=-G M_{\star} \boldsymbol{r} / r^{3}$ is the gravitational acceleration, where $G$ is Newton's constant, $M_{\star}$ is the mass of the star, $\boldsymbol{\Omega}_{0}=(\cos \theta,-\sin \theta, 0) \Omega_{0}$ is the rotation vector, $v$ is the kinematic viscosity, $\eta$ is the magnetic diffusivity, both assumed to be constant, $\boldsymbol{F}^{\mathrm{rad}}=-K \nabla T$ and $F^{\mathrm{SGS}}=-\chi_{\mathrm{SGS}} \rho T \nabla s$ are the radiative and subgrid scale (SGS) heat fluxes, where $K$ is the radiative heat conductivity and $\chi_{\mathrm{SGS}}$ is the turbulent heat conductivity, which represents the unresolved convective transport of heat, $s$ is the specific entropy, $T$ is the temperature, and $p=(\gamma-1) \rho e$ is the pressure, where $\gamma=c_{\mathrm{P}} / c_{\mathrm{V}}=5 / 3$ is the ratio of specific heats at constant pressure and volume, respectively, and $e=c_{\mathrm{V}} T$ is the specific internal energy. The rate-of-strain tensor $\mathbf{S}$ is given by $\mathrm{S}_{i j}=(1 / 2)\left(u_{i ; j}+u_{j ; i}\right)-(1 / 3) \delta_{i j} \boldsymbol{\nabla} \cdot \boldsymbol{u}$, where the semicolons denote covariant differentiation (Mitra et al. 2009).

\subsection{Initial and Boundary Conditions}

As in Käpylä et al. (2012), the initial state is isentropic, we fix the value of $\partial T / \partial r$ on the lower boundary, and choose $K$ to be proportional to $r^{-15}$ (Käpylä et al. 2013) such that convection is responsible for the majority of the energy transport. The density obeys hydrostatic equilibrium. We use a weak, random Gaussian noise, small-scale seed magnetic field.

The radial and latitudinal boundaries are assumed to be impenetrable and stress free, see Equations (8) and (9) of Käpylä et al. (2013). The magnetic field obeys perfect conductor conditions on $\theta=\theta_{0}$ and $\pi-\theta_{0}$ and $r=r_{0}$, and is radial on $r=R$, allowing magnetic helicity to escape the domain without accumulating it at the bottom of the convection zone; see Equations (10)-(12) of Käpylä et al. (2013). On the latitudinal boundaries we assume that density and entropy have vanishing first derivatives. By choosing the value of $\theta_{0}$ small enough, we hope to minimize the possibility of artifacts, which might be the case when the flow inside the inner tangent cylinder is fully included (Käpylä et al. 2012); see also Mitra et al. (2009) for comparative results varying the value of $\theta_{0}$. On the upper boundary we apply a black body condition

$$
\sigma T^{4}=-K \frac{\partial T}{\partial r}-\chi_{\mathrm{SGS}} \rho T \frac{\partial s}{\partial r},
$$

where $\sigma$ is a modified Stefan-Boltzmann constant (see Käpylä et al. 2013; Barekat \& Brandenburg 2013).

\subsection{Dimensionless Parameters}

As in Käpylä et al. (2013), we define our simulations by imposing the energy flux at the bottom boundary, $F_{\mathrm{b}}=-\left.(K \partial T / \partial r)\right|_{r=r_{0}}$ and the values of $\Omega_{0}, v, \eta$, and $\bar{\chi}_{\mathrm{SGS}}=\chi_{\mathrm{SGS}}\left(r_{\mathrm{m}}=0.85 R\right)$. The corresponding nondimensional input parameters are the luminosity parameter $\mathcal{L}=L_{0} /\left[\rho_{0}(G M)^{3 / 2} R^{1 / 2}\right]$ where $L_{0}=4 \pi r_{0}^{2} F_{\mathrm{b}}$, and the (turbulent) fluid and magnetic Prandtl numbers $\operatorname{Pr}=v / \bar{\chi}_{\mathrm{SGS}}$ and $\operatorname{Pm}=v / \eta$, and the non-dimensional viscosity $\tilde{v}=v / \sqrt{G M R}$. The density stratification is controlled by the normalized pressure scale height at the surface, $\xi=\left[(\gamma-1) c_{\mathrm{V}} T_{1}\right] /(G M / R)$.

Other useful diagnostic parameters are the fluid and magnetic Reynolds numbers $\mathrm{Re}=u_{\mathrm{rms}} /\left(\nu k_{\mathrm{f}}\right)$ and $\mathrm{Rm}=u_{\mathrm{rms}} /\left(\eta k_{\mathrm{f}}\right)$, where $k_{\mathrm{f}}=2 \pi / \Delta r \approx 21 R^{-1}$ is an estimate of the wavenumber of the largest eddies, and $\Delta r=R-r_{0}=0.3 R$ is the thickness of the layer. The Coriolis number is defined as Co $=2 \Omega_{0} \tau_{\text {to }}$, where $\tau_{\text {to }}=\left(u_{\text {rms }} k_{\mathrm{f}}\right)^{-1}$ is the turnover time and $u_{\mathrm{rms}}=\sqrt{(3 / 2)\left\langle u_{r}^{2}+u_{\theta}^{2}\right\rangle_{r \theta \phi \Delta t}}$ is the rms velocity and the subscripts indicate averaging over $r, \theta, \phi$, and a time interval $\Delta t$ of several magnetic diffusion times during which the run is thermally relaxed. We express the magnetic field in equipartition field strengths, $B_{\mathrm{eq}}(r)=\left\langle\mu_{0} \rho \boldsymbol{u}^{2}\right\rangle_{\theta \phi \Delta t}^{1 / 2}$, where all three components of $\boldsymbol{u}$ are included. We average over the $\phi$-coordinate to define mean quantities, denoted by an overbar. Furthermore, we define magnetic and kinetic energies as $E_{\text {mag }}=\left\langle\boldsymbol{B}^{2} / 2 \mu_{0}\right\rangle_{r \theta \phi \Delta t}$ and $E_{\text {kin }}=\left\langle\rho \boldsymbol{u}^{2} / 2\right\rangle_{r \theta \phi \Delta t}$, and denote the energies of the axisymmetric and $m=1$ modes of the magnetic field as $E_{\text {mag }}^{(0)}$ and $E_{\text {mag }}^{(1)}$, respectively. The simulations were performed with the PENCIL CODE. ${ }^{5}$

\subsection{Relation to Stellar Parameters}

We calibrate our model with solar parameters. However, due to the compressible formulation of our model, using the real solar luminosity would lead to prohibitively large Rayleigh numbers making the numerical solutions infeasible. Thus we increase the luminosity in our model to bring the dynamical and acoustic timescales closer to each other (Käpylä et al. 2013). The luminosity in our models is roughly $10^{6}$ times greater than in the Sun. This means that the convective velocity is roughly 100 times greater than in the Sun and we need to increase $\Omega_{0}$ by the same factor to achieve the same rotational influence (i.e., the same value of $\mathrm{Co}$ ) as in the Sun. We denote this solar-calibrated value by $\tilde{\Omega}_{\odot}$. In our simulations the rotation rate is 2.7-6.7 times faster than this, see Table 1. For further

\footnotetext{
5 http://pencil-code.googlecode.com/
} 

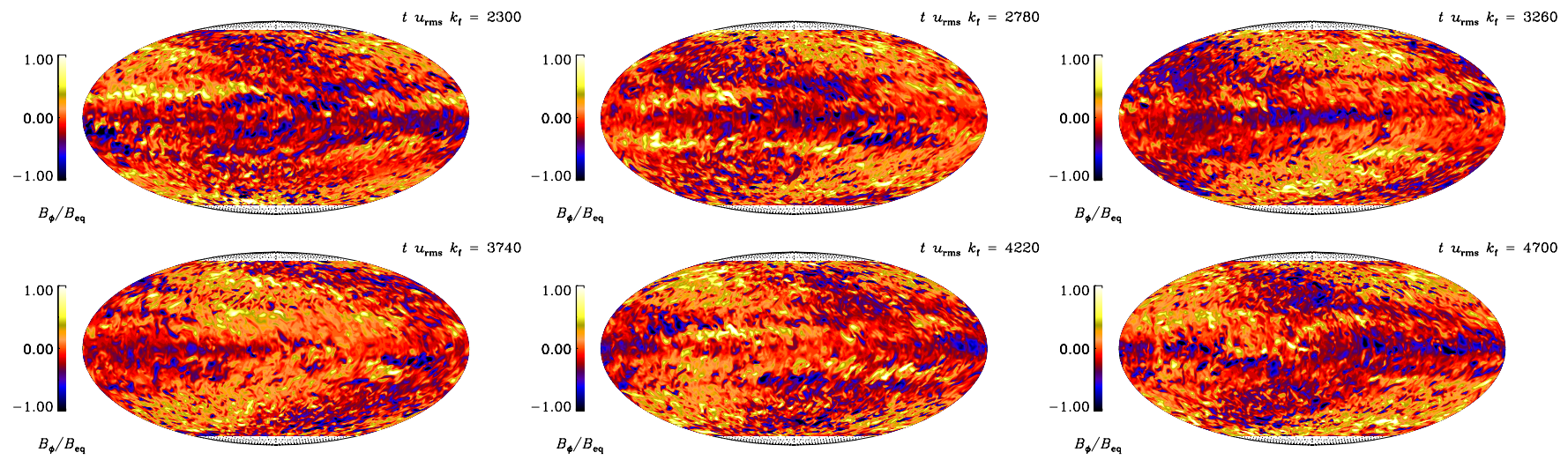

Figure 1. Azimuthal magnetic field $B_{\phi}$, normalized by the equipartition value $B_{\text {eq }}$, near the surface of the star at $r=0.98 R$ from Run B for six times separated by $480 \tau_{\text {to }}$.

(A color version of this figure is available in the online journal.)

Table 1

Summary of the Runs

\begin{tabular}{|c|c|c|c|c|c|c|c|c|c|c|c|c|}
\hline Run & $\operatorname{Pr}_{\mathrm{SGS}}$ & $\tilde{v}$ & $\mathrm{Ra}_{\mathrm{t}}$ & $\operatorname{Re}$ & $\mathrm{Rm}$ & $\mathrm{Co}$ & $\tilde{\Omega} / \tilde{\Omega}_{\odot}$ & $\tilde{E}_{\text {mag }}$ & $\tilde{E}^{(0)}$ & $\tilde{E}^{(1)}$ & $M$ & $P$ \\
\hline $\bar{A}$ & 3.5 & $4.1 \times 10^{-5}$ & $1.7 \times 10^{6}$ & 26 & 26 & 5.0 & 2.7 & 0.312 & 0.166 & 0.047 & 0.834 & -0.333 \\
\hline B & 3.0 & $3.5 \times 10^{-5}$ & $2.2 \times 10^{6}$ & 28 & 28 & 8.1 & 4.0 & 0.618 & 0.109 & 0.071 & 0.891 & 0.318 \\
\hline $\mathrm{C}$ & 3.0 & $3.5 \times 10^{-5}$ & $2.6 \times 10^{6}$ & 24 & 24 & 15.5 & 6.7 & 0.937 & 0.056 & 0.091 & 0.944 & 0.347 \\
\hline
\end{tabular}

Notes. Grid size is $128 \times 256 \times 512, \mathrm{Pm}=1, \xi=0.02, \mathcal{L}=3.8 \times 10^{-5}$, and $\tilde{\sigma}=\sigma R^{2} T_{0}^{4} / L_{0}=1.4 \times 10^{3}$, where $T_{0}=T\left(r_{0}\right)$. Furthermore, $\tilde{E}_{\mathrm{mag}}=E_{\mathrm{mag}} / E_{\mathrm{kin}}$, $\tilde{E}^{(0)}=E^{(0)} / E_{\text {mag }}$ and $\tilde{E}^{(1)}=E^{(1)} / E_{\mathrm{mag}} . M$ and $P$ characterize axial and equatorial symmetries; see Section 3.1.

explanation of our choice of parameters and the definition of the turbulent Rayleigh number $\mathrm{Ra}_{\mathrm{t}}$ from the thermally relaxed state, see Käpylä et al. (2013).

\section{RESULTS}

We discuss three simulations that can be interpreted as representing the Sun at younger ages when it was rotating more rapidly. Runs A, B, and C are respectively like Runs B3m, B4m, and B5m of Käpylä et al. (2012) where $\phi_{0}=\pi / 2$, but are now performed with a full $2 \pi$ azimuthal extent and $20-40$ per cent higher viscosity and magnetic diffusivity. Run B is equivalent to Run E4 of Käpylä et al. (2013) and was only run for $5500 \tau_{\text {to }}$. We run the simulations from the initial conditions discussed in Section 2.1 .

\subsection{Nonaxisymmetric Magnetic Fields}

We find that in the early stages of the simulations an axisymmetric oscillatory large-scale magnetic field grows first. This large-scale component shows equatorward migration for the two highest rotation rates (Käpylä et al. 2012, 2013). In the later stages, the dynamo mode changes into a stable nonaxisymmetric one, where the oscillations of the axisymmetric part cease (see Figure 17 of Käpylä et al. 2013).

Figure 1 shows a sequence of snapshots of $B_{\phi}$ from Run B near the surface at six times separated by $480 \tau_{\text {to }}$. Strong magnetic fields occur as extended belts of toroidal field near the equatorial region. These resemble the wreaths reported by Brown et al. (2010) and Nelson et al. (2013), but instead of predominantly axisymmetric structures, we now observe sign changes in longitude. Strong magnetic fields are also generated at higher latitudes. These structures appear to have a predominantly nonaxisymmetric distribution with a large negative radial magnetic field on one side with a positive counterpart on the other.

We Fourier filter the simulation data to extract the lowest order $m=0,1$ contributions to the magnetic field. We find that the energy of the nonaxisymmetric $m=1$ mode is of the same order of magnitude as the axisymmetric one in all runs (see Table 1), but observe a growth of the $m=1$ mode with respect to $m=0$ as rotation increases; see the increase of $E_{\text {mag }}^{(1)}$ combined with a decrease of $E_{\mathrm{mag}}^{(0)}$ in Figure 18 of Käpylä et al. (2013) for Run B. The lowest order modes constitute only roughly a fifth of the total magnetic field energy, the rest being in still higher $(m>1)$ modes.

We quantify the non-axisymmetry of the magnetic field with the quantities (see Rädler et al. 1990)

$$
M=1-\frac{E_{\mathrm{mag}}^{(0)}}{E_{\mathrm{mag}}}, \quad M^{(1)}=1-\frac{E_{\mathrm{mag}}^{(0)}}{E_{\mathrm{mag}}^{(0)}+E_{\mathrm{mag}}^{(1)}} .
$$

The random noise used as our initial condition yields $M \approx$ $M^{(1)} \approx 1$. A turbulent $m=0$ dynamo yields small values of $M^{(1)}=0$, but, owing to contributions from random noise to high $m$ modes, $M=O(1)$, while for a turbulent $m=1$ mode we have again $M \approx M^{(1)} \approx 1$. We show the time evolution of $M$ and $M^{(1)}$ in Figures 2(a) and (b). We find that $M$ is close to unity in the saturated stages of our runs. In earlier stages where the axisymmetric dynamo mode is more prominent, the minimum values of $M$ are between 0.6 and 0.7 , whereas $M^{(1)}$ can be as low as 0.1 . The ratio of the nonaxisymmetric to axisymmetric field components is not completely constant over time even in the saturated state, with variations of roughly 10 percent in comparison to the average values. Furthermore, the larger the rotation rate, the closer the solution is to a purely nonaxisymmetric solution. We quantify the equatorial symmetry of 

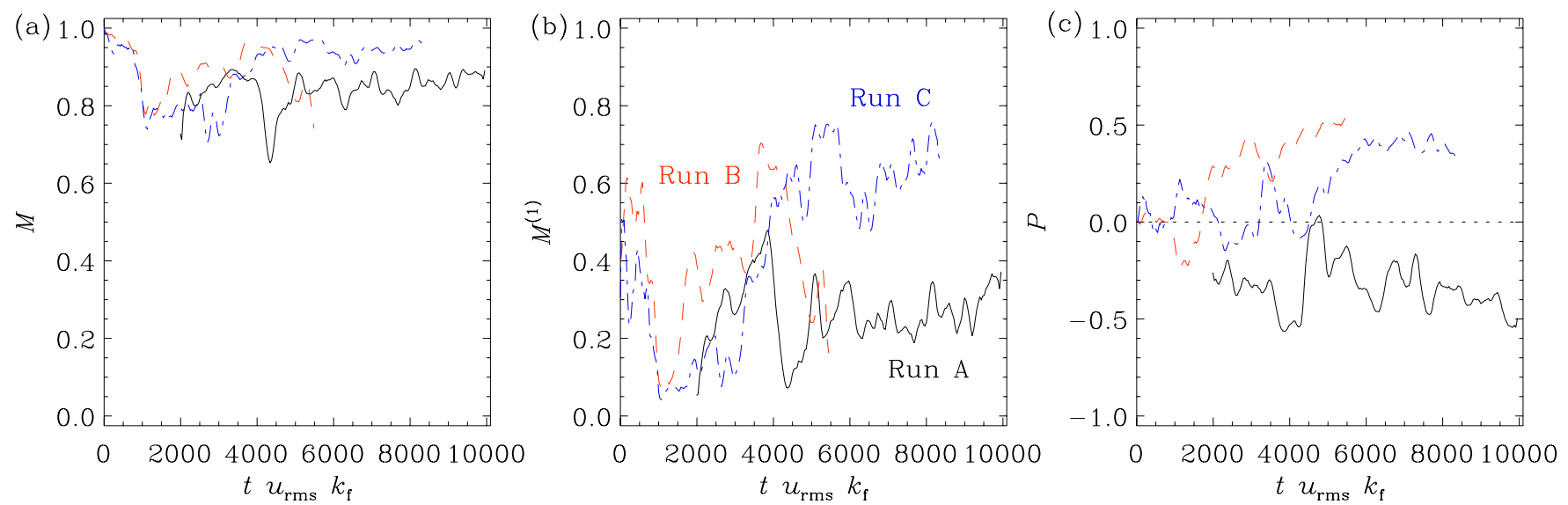

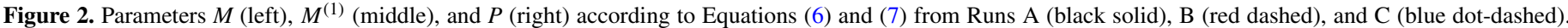
The data for Run A does not start from zero due to a lack of diagnostics output from the early part of the run.

(A color version of this figure is available in the online journal.)

the magnetic field by the parity (Brandenburg et al. 1989)

$$
P=\frac{E^{(\mathrm{S})}-E^{(\mathrm{A})}}{E^{(\mathrm{S})}+E^{(\mathrm{A})}}
$$

where $E^{(\mathrm{S})}$ and $E^{(\mathrm{A})}$ correspond to volume averaged energies of the symmetric and antisymmetric parts of the magnetic field. The extrema $P=1$ and $P=-1$ correspond to complete symmetry and complete anti-symmetry with respect to the equator. A random initial field produces $P=0$. As is apparent from Figure 2(c), there is mixed equatorial symmetry at all times in all of the runs. The lowest rotation case, Run A, persistently and preferentially shows an antisymmetric configuration, while Runs $B$ and $C$ with increased rotation evolve toward a symmetric configuration. This is a trend that is also seen in nonlinear, nonaxisymmetric mean-field (Rädler et al. 1990; Moss et al. 1995) and convection (Ishihara \& Kida 2002) models.

\subsection{Pattern Speed of the $m=1$ Structure}

Visual inspection of Figure 1 already reveals that the largescale non-axisymmetric structure is propagating in the retrograde direction in the frame rotating with the star. To analyze this drift quantitatively, we begin by using the Fourier-filtered data at the surface of the star at $r / R=1$. We then track the magnetic and temperature structures by following the extrema of $B_{r}$ and $T$ of the filtered (sinusoidal) signal. We measure the azimuth $\varphi$ of the resulting $m=1$ structure, and compute the pattern speed as $\Omega_{\text {pat }}=d \varphi / d t$. The resulting tracks of the magnetic extrema and corresponding temperature maxima from Run $\mathrm{B}$ are plotted in Figure 3 at different latitudes, together with the phase of the differential rotation measured from the flow. The signal in the magnetic field is well visible in both hemispheres, while that in temperature is more clear in the southern hemisphere. For this reason, we perform the analysis only for the southern hemisphere.

From Figure 3 it is evident that the nonaxisymmetric structure is moving in the retrograde direction with nearly constant speed. The pattern speed is considerably slower than the one expected from advection by differential rotation at any latitude. The $m=1$ structure completes an orbit in roughly $2400 \tau_{\text {to }}$, whereas
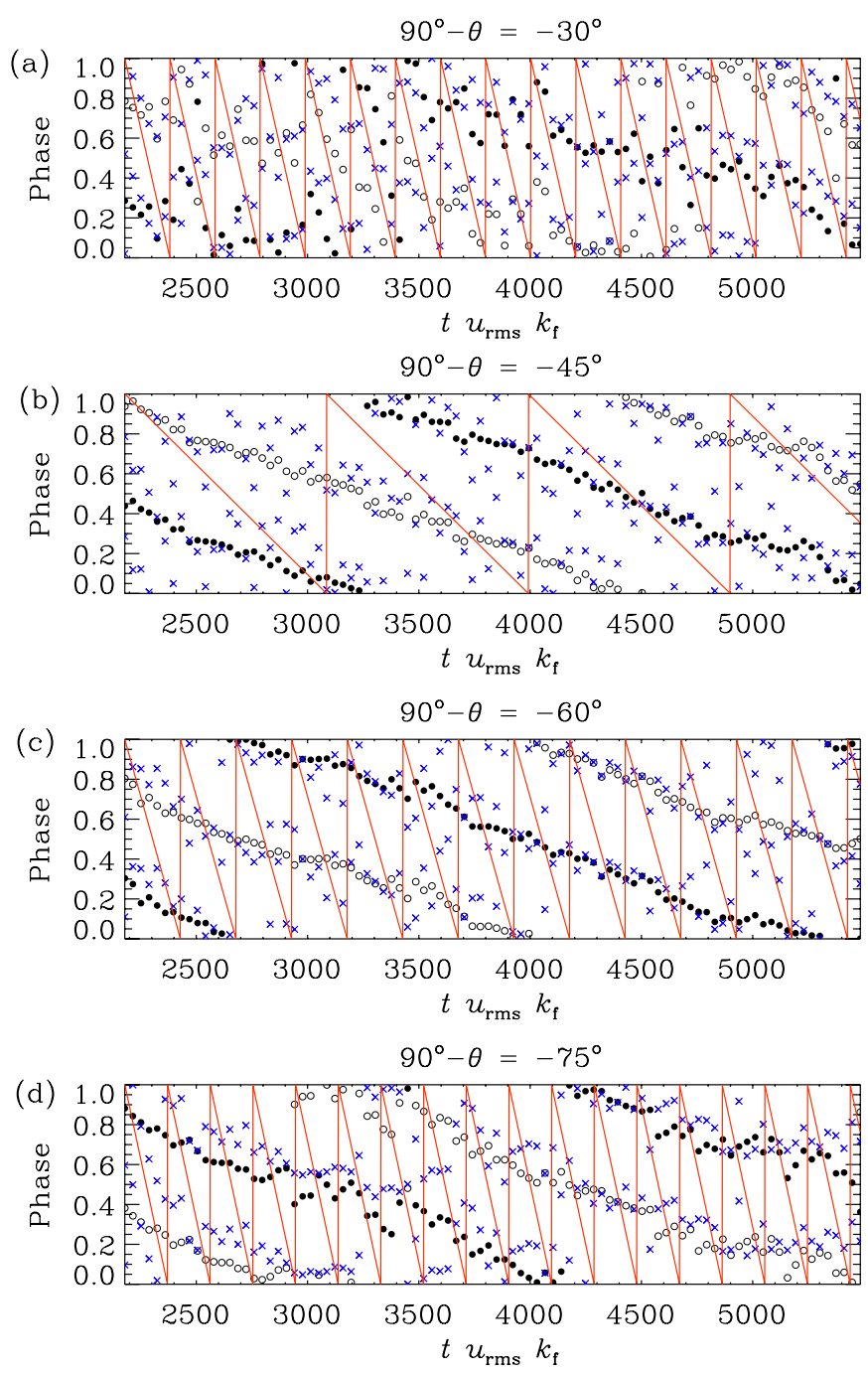

Figure 3. Phase of the $m=1$ mode of $B_{r}$ (filled and open circles) and the $m=2$ mode of $T$ (blue crosses) at the surface as a function of time from Run B at four latitudes. Red lines denote the phase based on pure advection due to differential rotation.

(A color version of this figure is available in the online journal.) 

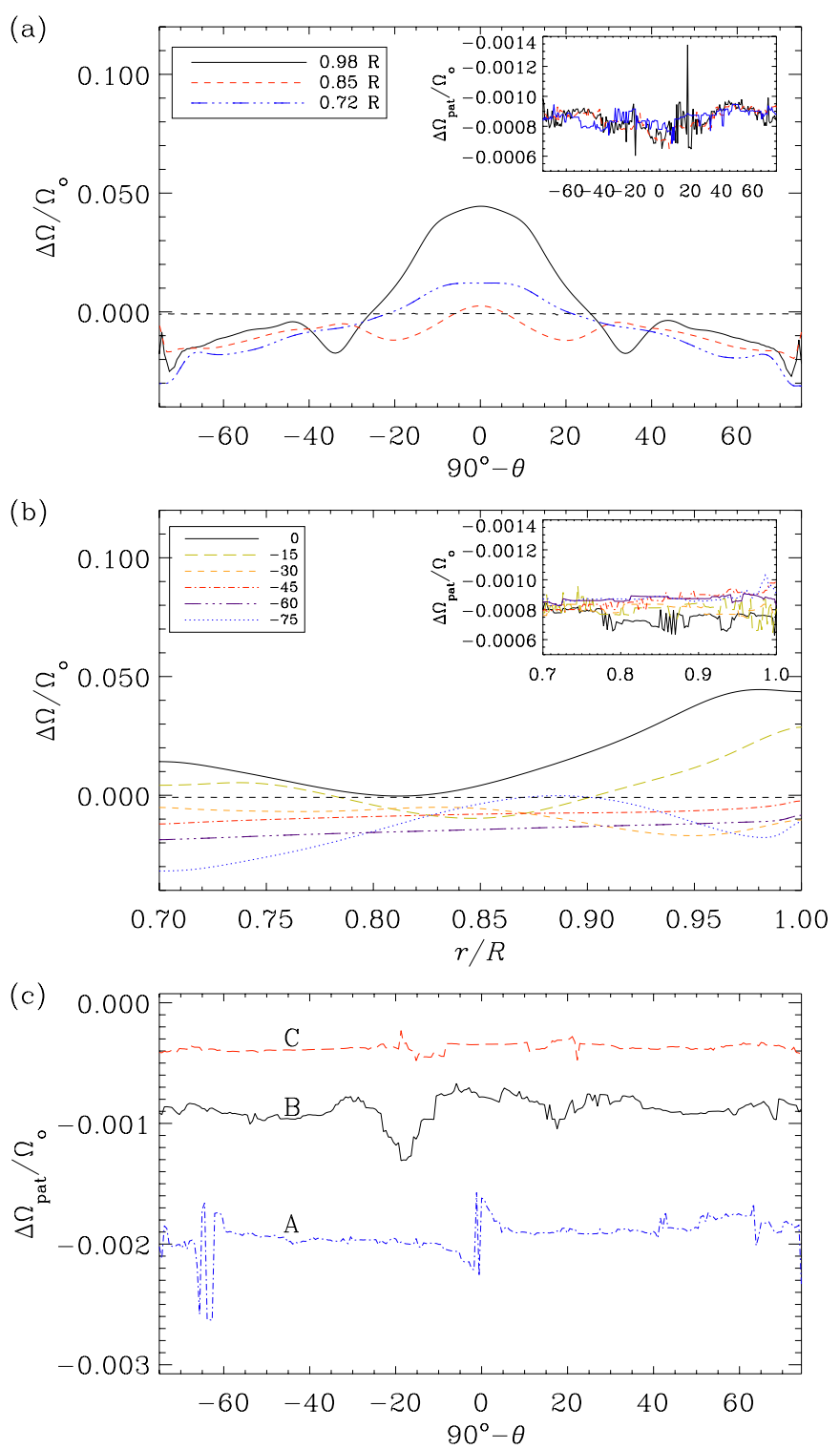

Figure 4. Top: time-averaged differential rotation $\Delta \Omega / \Omega_{0}=\bar{u}_{\phi} / \Omega_{0} r \sin \theta-1$ from different depths and from the non-axisymmetric structure (black dashed horizontal line, enlarged in inset) during the saturated state. Middle: angular velocity as a function of depth at select latitudes. Bottom: phase speed of the pattern, $\Omega_{\text {pat }}=d \varphi / d t$ from Runs A, B, and C.

(A color version of this figure is available in the online journal.)

pure advection due to differential rotation is typically 5-10 times faster. From the analysis of the magnetic field and temperature at the surface, it is evident that the nonaxisymmetric structure rotates without being affected by differential rotation.

\subsection{Relation to Local Rotation Rate}

Next we analyze the situation more thoroughly by computing the rotation profile of the nonaxisymmetric mode $m=1$ from all the runs as functions of latitude and depth, and compare it to the differential rotation profiles, see Figure 4. While the gas shows more differential rotation near the surface, the $m=1$ pattern is essentially rigidly rotating; see Figure 4(a). In Figure 4(b) we show the radial dependence of gas and pattern speeds. The pattern speed is retrograde by $0.09 \%$, showing a very small positive radial gradient near the equator in the deeper part. In all of these cases we find that the magnetic structure is propagating in the retrograde direction, see Figure 4(c). The normalized pattern speed $\Delta \Omega_{\text {pat }}=\Omega_{\text {pat }} / \Omega_{0}$ is monotonically decreasing as a function of rotation rate. Our analysis, therefore, shows that the almost rigid pattern speed $\Omega_{\text {pat }}$ does not match the differential rotation of the star at any depth or latitude.

We find a corresponding signal in the temperature at high latitudes; see Figure 3. However, the maxima of $\boldsymbol{B}^{2}$ correspond to the temperature maxima rather than the minima. Temperature fluctuations $\Delta \bar{T} / \bar{T}$ of the $m=2$ mode are 2-5 percent. These fluctuations are largely independent of the normalized radial component of the magnetic field, $\bar{B}_{r} / B_{\text {eq. }}$. This differs from the results of Run C1 from Käpylä et al. (2013) where fluctuations were between 15-20 percent.

\section{CONCLUSIONS}

We have studied azimuthal dynamo waves in threedimensional simulations of convection-driven dynamos. The wave moves rigidly and is generally slower than the differentially rotating gas. The drift cannot be accounted for by the differential rotation at any depth in the simulations. In the parameter regime investigated, only non-cyclic solutions with retrograde patterns were found, and their speed decreases with increasing rotation. In all of the cases investigated, the nonaxisymmetric pattern makes one orbit in the co-rotating frame in a few thousand convective turnover times, 5-10 times slower than expected if differential rotation of the fluid was the cause. This is compatible with the behavior of nonaxisymmetric dynamo modes discussed in Section 1; see Rädler (1986).

In active rapid rotators, the reported pattern speeds are of the same order (in absolute terms) as reported in this study, see Lindborg et al. (2013). The deduced amounts of differential rotation for these objects (see, e.g., Henry et al. 1995; Marsden et al. 2005; Siwak et al. 2010), however, are much weaker than those obtained in Runs A-C. As a result, in real objects the pattern speeds of non-axisymmetric structures are only slightly smaller than or comparable to what is expected from advection by differential rotation. Also, both prograde and retrograde drifts have been observed (see, e.g., Berdyugina \& Tuominen 1998), and commonly the patterns are disrupted (see, e.g., Hackman et al. 2013), the longest reported drift so far being of the order of a decade (Berdyugina \& Tuominen 1998). The latitudeindependent drift of the spot structure in II Peg reported by Lindborg et al. (2013), however, is not consistent with latitudedependent differential rotation and is more consistent with the results presented here. In real objects, however, the dynamo seems to operate in a regime where the azimuthal dynamo wave and differential rotation have similar pattern speeds and these two mechanisms compete with each other. Preliminary studies suggest that such behavior could be obtained at larger rotation speeds, and will be discussed in a future publication.

We thank Thomas Hackman for his comments on the manuscript. The simulations were performed using the supercomputers hosted by CSC-IT Center for Science Ltd. in Espoo, Finland, who are administered by the Finnish Ministry of Education. Financial support from the Academy of Finland grants No. 136189, 140970 (P.J.K.), and 218159, 141017 (M.J.M.), as well as the Swedish Research Council grants 621-2011-5076 and 2012-5797, and the European Research Council under the AstroDyn Research Project 227952 are acknowledged. The authors thank NORDITA for hospitality during their visits. 


\section{REFERENCES}

Augustson, K., Brun, A. S., Miesch, M. S., \& Toomre, J. 2013, arXiv:1310.8417 Barekat, A., \& Brandenburg, A. 2013, A\&A, submitted (arXiv:1308.1660) Berdyugina, S. V., \& Tuominen, I. 1998, A\&A, 336, L25

Brandenburg, A. 2007, in IAU Symp. 239, Convection in Astrophysics, ed. F. Kupka, I. Roxburgh, \& K. L. Chan (Cambridge: Cambridge Univ. Press), 457

Brandenburg, A., Krause, F., Meinel, R., Moss, D., \& Tuominen, I. 1989, A\&A 213, 411

Brown, B. P., Browning, M. K., Brun, A. S., Miesch, M. S., \& Toomre, J 2010, ApJ, 711, 424

Brown, B. P., Miesch, M. S., Browning, M. K., Brun, A. S., \& Toomre, J. 2011, ApJ, 731, 69

Busse, F. H. 2004, Chaos, 14, 803

Busse, F. H. 2007, SoPh, 245, 27

Gastine, T., Duarte, L., \& Wicht, J. 2012, A\&A, 546, A19

Ghizaru, M., Charbonneau, P., \& Smolarkiewicz, P. K. 2010, ApJL, 715, L133

Gizon, L., Duvall, T. L., \& Schou, J. 2003, Natur, 421, 43

Goudard, L., \& Dormy, E. 2008, EL, 83, 59001

Green, C. A., \& Kosovichev, A. G. 2006, ApJL, 641, L77

Hackman, T., Mantere, M. J., Lindborg, M., et al. 2012, A\&A, 538, A126

Hackman, T., Pelt, J., Mantere, M. J., et al. 2013, A\&A, 553, A40

Henry, G. W., Eaton, J. A., Hamer, J., \& Hall, D. S. 1995, ApJS, 97, 513

Ishihara, N., \& Kida, S. 2002, FlDyR, 31, 253

Jetsu, L., Pelt, J., \& Tuominen, I. 1993, A\&A, 278, 449

Käpylä, P. J., Korpi, M. J., \& Brandenburg, A. 2009, A\&A, 500, 633
Käpylä, P. J., Korpi, M. J., Brandenburg, A., Mitra, D., \& Tavakol, R. 2010, AN 331, 73

Käpylä, P. J., Mantere, M. J., \& Brandenburg, A. 2012, ApJL, 755, L22

Käpylä, P. J., Mantere, M. J., Cole, E., Warnecke, J., \& Brandenburg, A. 2013, ApJ, 778, 41

Kitchatinov, L. L., \& Rüdiger, G. 1999, A\&A, 344, 911

Kochukhov, O., Mantere, M. J., Hackman, T., \& Ilyin, I. 2013, A\&A, 550, A84

Korhonen, H., Berdyugina, S. V., Hackman, T., et al. 2007, A\&A, 476, 881

Lindborg, M., Mantere, M. J., Olspert, N., et al. 2013, A\&A, 559, A97

Marsden, S. C., Carter, B. D., \& Donati, J.-F. 2005, in 13th Cambridge Workshop on Cool Stars, Stellar Systems and the Sun, ed. F. Favata, G. A. J. Hussain, \& B. Battrick (ESA Special Publication, Vol. 560; Noordwijk: ESA), 799

Mitra, D., Tavakol, R., Brandenburg, A., \& Moss, D. 2009, ApJ, 697, 923

Moss, D., Barker, D. M., Brandenburg, A., \& Tuominen, I. 1995, A\&A, 294, 155

Nelson, N. J., Brown, B. P., Brun, A. S., Miesch, M. S., \& Toomre, J. 2013, ApJ, 762,73

Ossendrijver, M. 2003, A\&ARv, 11, 287

Pulkkinen, P., \& Tuominen, I. 1998, A\&A, 332, 748

Rädler, K.-H. 1986, AN, 307, 89

Rädler, K.-H., Wiedemann, E., Brandenburg, A., Meinel, R., \& Tuominen, I. 1990, A\&A, 239, 413

Rüdiger, G. 1978, AN, 299, 217

Siwak, M., Rucinski, S. M., Matthews, J. M., et al. 2010, MNRAS, 408, 314

Warnecke, J., Käpylä, P. J., Mantere, M. J., \& Brandenburg, A. 2013, ApJ, 778,141 